\title{
Corruption and Supervision Costs in Hierarchies ${ }^{1}$
}

\author{
Mehmet BaC \\ Bilkent University, Bilkent, Ankara, Turkey 06533
}

Received December 8, 1994; revised January 12, 1996

Bac, Mehmet - Corruption and Supervision Costs in Hierarchies

We study the problem of organizing three agents in a hierarchical monitoring structure and designing a corresponding incentive system to minimize the cost of implementing a target level of corruption. We show that the possibility of collusion may prevent the implementation of anything less than full corruption. In relatively flat hierarchies, economies of scale in monitoring reduce implementation costs but may increase the risk of collusion. We contrast the performance of the hierarchy where one supervisor monitors two subordinates with the supervision chain, whose upper part is shown to display a higher risk of collusion than its lower part. J. Comp. Econom., April 1996, 22(2), pp. 99-118. Bilkent University, Bilkent, Ankara, Turkey 06533. (c) 1996 Academic Press, Inc.

Journal of Economic Literature Classification Numbers: D73, K42.

\section{INTRODUCTION}

The design of a hierarchical organization and a corresponding incentive system must prevent corrupt behavior at the individual level without causing collusion between supervisors and subordinates. The potential conflict between the goals of preventing corruption and collusion has recently been studied in formal models that add a supervisor to the classical hierarchy consisting of a principal and an agent (pioneering papers are by Tirole (1986) and Laffont (1990)). The central theme in the growing literature that explores the principal-supervisor-agent paradigm is the impact of possible collusion

${ }^{1}$ The comments and suggestions of an anonymous referee have greatly improved the content and presentation of this paper. All remaining errors are mine. 
on contractual and institutional arrangements. ${ }^{2}$ The present paper builds on this literature. It extends the analysis to the problem of designing a hierarchical structure and a corresponding wage-reward scheme that minimizes the cost of implementing a target level of corruption in a group of three identical agents. We take the expected number of bribed agents as a proxy for the level of corruption. Assuming three agents is the simplest, yet sufficiently general, means of exploring the relative performance of steep and flat hierarchies in terms of their implementation costs and collusion possibilities.

We view hierarchies as collections of antisymmetric monitoring relations; that is, as trees depicting who supervises whom. Supervision is an activity that takes effort as input, producing a binary, random success variable. An important element of the analysis is thus the behavior of per-subordinate success probabilities with respect to the number of subordinates, the type of economies or diseconomies of scale in monitoring. Another important element is the distinction between external (individual) and internal (organized) corruption. Organization theorists have long discussed these two dimensions of corrupt behavior (e.g., Cyert and March, 1963), but the issue has only recently been recognized in the economics literature (see, for example, the papers by Tirole $(1986,1988)$ ). External corruption involves an independent and individual violation of duty, as typically exemplified by the bribe-taking public official. In contrast, internal corruption is an organized crime. It can take the form of a collusion whereby the subordinates transfer a portion of the proceeds from external corruption (bribes) to upper levels and obtain protection in return. An internally corrupt hierarchy has no supervisory function, and it will also display maximal external corruption because each member will act so as to maximize total bribe revenues that can be collected in the hierarchy. Whether the outcome will exhibit internal or just a limited level of external corruption depends of course on the structure of the hierarchy and incentives that the principal provides to the supervisor(s). The latter may be motivated by the promised reward for a successful bribe report and thus monitor effectively or exert authority to appropriate the subordinates' corrupt gains. These considerations raise several questions: Can the principal achieve the dual goal

${ }^{2}$ Kofman and Lawarré (1993) introduce an external supervisor and show that the possibility of collusion affects the optimal incentive system. They also show that sometimes the external/ internal supervisor will not be used as a response to possible collusion. Other related research include papers studying the impact of supervision on firm size and workers' incentives and those aiming at comparing hierarchies in terms of their efficiency in gathering and processing information. The supervisory role of hierarchies is studied by Williamson (1967), Calvo and Wellisz (1978), and Calvo (1987). Varian (1990) presents a recent treatment of supervision chains. As for the second line of research, examples include Radner (1993) who studies the problem of minimizing delay in processing and communicating information in hierarchies, and Baron and Besanko (1992) who present hierarchical contracts as solutions to the problem of maximizing a regulator's surplus under hidden information. Bolton and Dewatripont (1994) also explore the implications of information processing costs on firm size. 
of reducing external corruption to a target level at no risk of internal corruption? What form of hierarchy minimizes the members' surplus from internal corruption? Do economies of scale in the monitoring technology favor flat hierarchies, even with possible collusion? What about the cost of monetary incentives to achieve these goals?

In Section II we describe the model with which we address these questions. Next, we consider in Section III the basic hierarchy consisting of two agents, a supervisor and a subordinate, to display the workings of the model. We show that inducing effective monitoring brings in the risk of internal corruption. It may even be impossible to prevent bribe taking without causing collusion, especially if the agents' reservation wages are high and/or the size of a potential bribe is large. However, if implementing any probability of bribe acceptance is possible with risk-neutral agents and unbounded rewards to supervisors, any probability can be implemented at the same expected cost. When rewards are bounded from above, there is a lower bound on the expected implementable level of corruption.

We extend the analysis to three-agent hierarchies in Section IV. First, we derive the least-cost solution to implementing the target level of corruption in two different hierarchies, the supervision chain and a flat hierarchy where a supervisor monitors two agents. Larger economies of scale in the monitoring technology generate cost savings in the flat hierarchy which, in the absence of internal corruption, dominates the supervision chain if economies of scale are strong enough.

In the second part of Section IV we introduce the possibility of internal corruption and study its likelihood in the two hierarchies. Our analysis of the steep hierarchy, the supervision chain, shows that the total surplus from internal corruption is larger at the upper layers. This result is due to a monitoring externality in the supervision chain. Top supervisor's monitoring increases the middle supervisor's opportunity cost of accepting a bribe because the latter will not be rewarded for documenting the bottom agent's corruption if he himself is proved corrupt. The principal exploits this monitoring externality to reduce implementation costs; he adjusts incentives to induce a lower monitoring effort on the top supervisor. As a result, the top supervisor's expected rewards from monitoring are lower and he can more easily be bribed to stop monitoring.

Finally, we focus on the relationship between the collusion-proofness constraint in the flat hierarchy and types of economies of scale in monitoring. We show that diseconomies of scale in the form of a proportional decrease in the per-subordinate detection probability reduces the members' surplus from internal corruption. Section $\mathrm{V}$ presents a summary and extensions for future research.

\section{THE MODEL}

Our model comprises four risk-neutral individuals, a principal and three identical agents. The reservation wage of the agents is $w$. The agents enjoy 


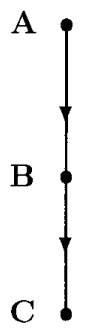

H1

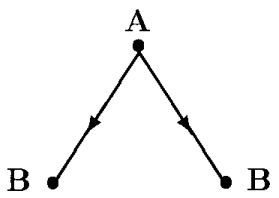

$\mathrm{H} 2$

FIGURE 1

some discretion in their job that they can misuse for private gain, not necessarily of a monetary kind. This potential for external corruption is represented in the model by letting each agent be offered a bribe of value $z$. Agent $i$ 's possibly mixed decision on whether to accept the bribe is denoted $b_{i} \in$ $[0,1]$, which takes the value $b_{i}=1$ for acceptance, and $b_{i}=0$ otherwise. We refer to $\sum b_{i}$ as the expected level of corruption in the group. The best example to motivate the analysis is a public office where the agents are officials and the principal is the government. We can also think of the agents as employees of a firm, where bribe should be interpreted as on-the-job consumption that the employees offer themselves.

The principal has to design a hierarchical monitoring structure in order to keep corruption under control. By hierarchical monitoring, we mean a set of antisymmetric monitoring relations implied naturally by the form of the hierarchy. Antisymmetry in monitoring rules out reciprocal monitoring; that is, Agent B cannot monitor Agent A if A monitors B. We say that A is one rank above $\mathrm{B}$ if $\mathrm{A}$ monitors $\mathrm{B}, \mathrm{A}$ is two ranks above $\mathrm{B}$ if $\mathrm{A}$ monitors an agent who monitors $\mathrm{B}$, and so on.

We consider two hierarchical structures to organize three agents (see Fig. 1). The steepest hierarchy, denoted $H 1$, consists of a supervision chain where Agent A monitors Agent B, who in turn monitors Agent C. The second hierarchy, $H 2$, has Agent A monitoring the other two agents, B and $\mathrm{C} .^{3}$ The set of possible hierarchies is denoted $H=\{H 1, H 2\}$.

An agent who is proved corrupt gets a utility normalized to zero. Furthermore, to simplify the analysis, we will assume that it is completely safe to

${ }^{3}$ The completely decentralized structure where each agent is an independent unit is excluded from our analysis because it has no supervisory function. It displays the maximum level of corruption. On the other hand, the hierarchy that leaves Agent $\mathrm{C}$ unmonitored and monitors Agent B through Agent A is uninteresting because it is strictly dominated by $H 1$ (any level of corruption can be implemented in $H 1$ at a lower cost). 
accept bribes in the absence of monitoring. ${ }^{4}$ Then, the expected utility of an agent who neither monitors nor is monitored is $E U_{i}=b_{i}\left(w_{i}+z\right)+(1-$ $\left.b_{i}\right) w_{i}$ which, not surprisingly, shows that the dominant strategy is to accept the bribe.

The costs involved in monitoring are described by the function $c(\mathrm{~nm})$, where $m$ denotes the per-subordinate monitoring effort and $n=1,2$ is the number of subordinates. Success is stochastic: a higher monitoring effort increases the per-subordinate probability $\mu(n m, n)$ of successfully detecting and documenting the bribe. In the case of one subordinate, we simply use $\mu(m)$ to denote $\mu(m, 1)$. We make the following standard assumption:

$c(n m)$ is increasing and strictly convex, and $\mu(n m, n)$ is increasing and strictly concave in $m$, with $\mu(n m, n) \rightarrow 1$ as $m \rightarrow \infty$. Furthermore, $c(0)=\mu(0, n)=0$, and $\mu^{\prime}(n m, n) \rightarrow \infty$ and $c^{\prime}(n m) \rightarrow 0$ as $m \rightarrow 0$.

Assumptions about the behavior of $\mu(n m, n)$ with respect to $n=1,2$ will be introduced later in Section IV where we study $H 2$. Let $p_{i}$ denote the benefit or the monetary reward that the principal offers supervisor $i$ per successful bribe report. Then, if supervisor $i$ himself is not monitored and monitors all his subordinates with the same effort $m_{i}$, his expected rewards are $R_{i}\left(h, p_{i}\right.$, $t)=p_{i} \mu\left(n m_{i}, n\right) \Sigma_{j} b_{j}$, where $\Sigma_{j} b_{j}$ is the expected number of bribed subordinates, and $t$ is the target level of corruption per agent. The principal's objective is to minimize the cost, the wage bill plus expected reward payments, of implementing the corruption level $t$. To achieve this goal, he will choose rewards $\left\{p_{i}\right\}$, wages $\left\{w_{j}\right\}$, and a hierarchy $h$. The minimum cost corresponding to hierarchy $h$ is denoted $T C(h)$. We state and explain the principal's problem as

$$
\min _{h \in H, p_{i}, w_{j}} \sum_{i} R_{i}\left(h, p_{i}, t\right)+\sum_{j} w_{j}
$$

subject to

$$
\begin{aligned}
& b_{j}^{*} \in \operatorname{argmax}_{b_{j}}\left[E U_{j}\left(m_{i}^{*}, b_{j}\right)\right] \\
& m_{i}^{*} \in \operatorname{argmax}_{m_{i}}\left[E U_{i}\left(m_{i}, b_{j}^{*}\right)\right] \\
& \left(\sum_{n} b_{n}^{*}\right) / 3=t<1, \\
& E U_{i} \geqslant w, \quad i=1,2,3, \\
& N C_{i}\left(h, p_{i}, w_{j}, t\right) \geqslant 0 .
\end{aligned}
$$

${ }^{4}$ One can easily incorporate an exogenous probability of being caught while taking the bribe to represent the case where outsiders blow the whistle. This extension, however, has no qualitative impact on our results. 
$N C_{i}\left(h, p_{i}, w_{j}, t\right) \geqslant 0$ represents the no-collusion constraint, which eliminates internal corruption involving supervisor $i$ and his immediate subordinates. The form of the expression for $N C_{i}\left(h, p_{i}, w_{j}, t\right)$ may depend on the shape of the hierarchy $h$, on the corresponding wage-reward scheme, and on the target level of corruption. A hierarchical structure and a vector of rewards and wages $\left(h, p_{i}, w_{j}\right)$ generate monitoring games between supervisors and subordinates. We require that the strategies $\left(b_{j}^{*}, m_{i}^{*}\right)$ form a Nash equilibrium of these games, as stated in constraints (1) and (2). The problem above can thus be stated as minimizing the cost of Nash-implementing the target level of corruption through a hierarchical structure and corresponding wage-reward schemes. Equation (3) is the implementation constraint: the average expected level of corruption must be $t$. If the hierarchy displays internal corruption, however, $\sum b_{n}=3$ and the corruption target in (3) is missed, unless, of course, the target is a fully corrupt hierarchy. Finally, (4) requires the solution $\left(h^{*}\right.$, $\left.p_{i}^{*}, w_{j}^{*}\right)$ to satisfy the participation constraints.

\section{THE BASIC HIERARCHY}

We consider in this section a relatively simple implementation problem where the number of agents is two. Agent A will supervise Agent B. We refer to this monitoring structure as the basic hierarchy. The analysis of the basic hierarchy is a useful starting point to show the workings of the model and the interplay of supervision and internal and external corruption.

The wages $\left(w_{\mathrm{A}}, w_{\mathrm{B}}\right)$ and the reward $p>0$ for A's successful bribe report generate a monitoring game between A and B. A's strategy is a monitoring effort $m$ and B's strategy is a bribe acceptance probability $b$. The Nash equilibrium of this game is denoted $\left(m^{*}, b^{*}\right)$. The expected utilities and participation constraints of the two agents are

$$
\begin{aligned}
& E U_{\mathrm{A}}=w_{\mathrm{A}}+z+p b \mu(m)-c(m) \geqslant w, \\
& E U_{\mathrm{B}}=b(1-\mu(m))\left(w_{\mathrm{B}}+z\right)+(1-b) w_{\mathrm{B}} \geqslant w .
\end{aligned}
$$

In the expression for $E U_{\mathrm{A}}$, we take into account the fact that $\mathrm{A}$ will accept the bribe because he is not monitored and $z>0$. Assumption A1 implies a unique Nash equilibrium, where $m^{*}>0$ if $p>0$ and $b^{*} \neq 0$. In equilibria where $b^{*} \in(0,1)$, Agent $\mathrm{B}$ hesitates between accepting and rejecting the bribe, which means $\left(1-\mu\left(m^{*}\right)\right)\left(w_{\mathrm{B}}+z\right)=w_{\mathrm{B}}$, or

$$
\mu\left(m^{*}\right)=\frac{z}{z+w_{\mathrm{B}}} .
$$

The equilibrium condition in (1), which states that the monitoring effort should be optimal given the subordinate's bribe acceptance strategy, can now be written as 


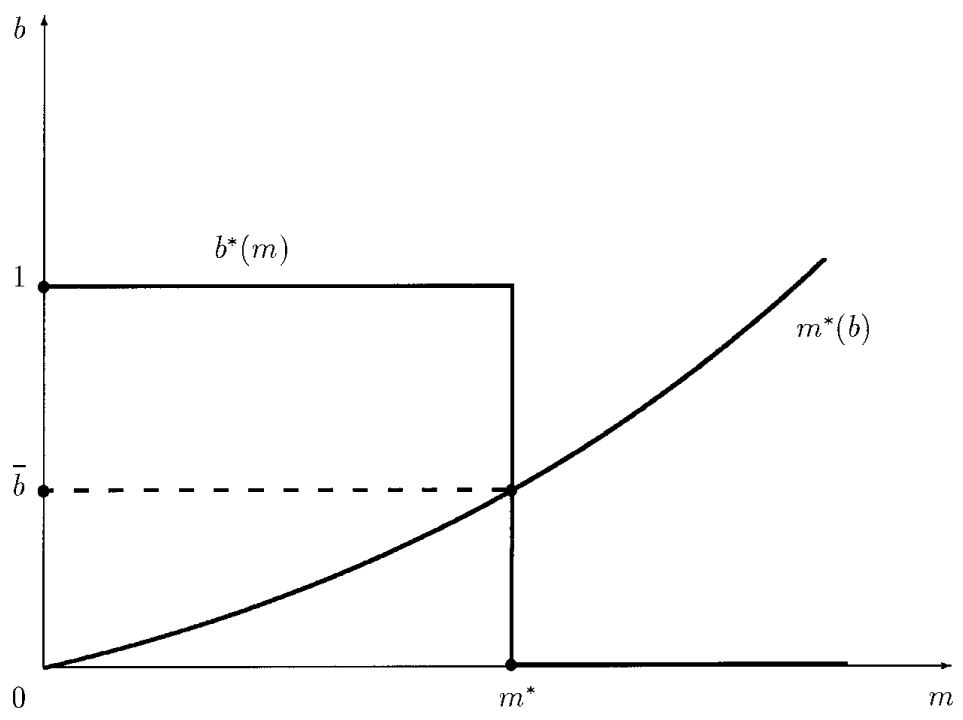

FIGURE 2

$$
p b^{*}=\frac{c^{\prime}\left(m^{*}\right)}{\mu^{\prime}\left(m^{*}\right)} .
$$

The intuition for why A's monitoring should make B indifferent can be grasped from Fig. 2, where $b^{*}(m)$ and $m^{*}(b)$ represent, respectively, B's and A's bestreply functions. There is a critical level of monitoring $m^{*}$, defined by (7), such that $\mathrm{B}$ rejects the bribe if $m>m^{*}$; however, then A's best reply is to stop monitoring, to which B replies optimally with $b=1$. If $m^{*}(1)<m^{*}$, we have an equilibrium involving a corner solution: Monitoring is positive but ineffective, i.e., B sets $b^{*}=1$ and accepts the bribe despite A's positive monitoring. Then, (7) becomes $\mu\left(m^{*}\right)<z /\left(z+w_{\mathrm{B}}\right)$, and (8) holds for $b^{*}=1$.

We can now state the principal's problem as

$$
\min _{p, w_{\mathrm{A}}, w_{\mathrm{B}}}\left[p b^{*} \mu\left(m^{*}\right)+w_{\mathrm{A}}+w_{\mathrm{B}}\right]
$$

subject to (5), (6), (7), (8), $b^{*}=\bar{b}$, and the no-collusion constraint, which we introduce later. We take $\bar{b} \in(0,1)$, because $\bar{b}=0$ cannot be implemented and implementation of $\bar{b}=1$ is trivial; set $p=0$ and choose wages to bind the participation constraints.

Before characterizing the least-cost solution, it will be useful to clarify the relationship between the Nash equilibrium $\left(m^{*}, b^{*}\right)$, total costs, and the three instruments of the principal $\left(p, w_{\mathrm{A}}, w_{\mathrm{B}}\right)$. Let us ignore the no-collusion constraint for the moment. Note that since $w_{\mathrm{A}}$ has no impact on the equilibrium 
conditions (7) and (8), the principal should set $w_{\mathrm{A}}$ as low as possible. A's participation constraint should thus be binding, which yields

$$
w_{\mathrm{A}}^{*}=w-z-p \bar{b} \mu\left(m^{*}\right)+c\left(m^{*}\right) .
$$

The choice of $w_{\mathrm{B}}$, on the other hand, presents a trade-off. From (7), we see that a lower $w_{\mathrm{B}}$ requires a higher equilibrium monitoring effort to implement a $\bar{b} \in(0,1)$; hence, the principal must increase $w_{\mathrm{A}}$ to compensate for A's higher cost of monitoring effort. Consequently, the principal may find it optimal to increase $w_{\mathrm{B}}$ above $w$ and economize on $w_{\mathrm{A}}$ by inducing a lower monitoring effort on A. Substitutions from (9) for $w_{\mathrm{A}}$ and from (7) for $w_{\mathrm{B}}$ in the expression of total costs yield

$$
w-z+c(m)+z \frac{(1-\mu(m))}{\mu(m)} .
$$

The principal's problem can thus be stated as to choose an effort level $m^{*}$ for A to minimize (10) subject to B's participation constraint. Given this $m^{*}$ and the target level of corruption $\bar{b}=b^{*}, p^{*}$ and $w_{\mathrm{B}}^{*}$ will be determined through (8) and (7). The cost-minimizing $m^{*}$ is determined through the firstorder condition

$$
c^{\prime}\left(m^{*}\right)=z \frac{\mu^{\prime}\left(m^{*}\right)}{\left[\mu\left(m^{*}\right)\right]^{2}},
$$

provided that it also satisfies the participation constraint $w \leqslant z\left(1-\mu\left(m^{*}\right)\right)$ / $\mu\left(m^{*}\right)=w_{\mathrm{B}}^{*}$. The first-order condition above corresponds to an interior solution $w_{\mathrm{B}}^{*} \geqslant w$ and has a natural interpretation: the marginal cost of effort, which the principal incurs by compensating A through (9), should be equal to its marginal benefit, which the principal enjoys by reducing $w_{\mathrm{B}}$ through (7). Otherwise, if $w>z\left(1-\mu\left(m^{*}\right)\right) / \mu\left(m^{*}\right)$, we have a corner solution $w_{\mathrm{B}}^{*}$ $=w$ (B's participation constraint is binding) and $m^{*}$ is determined through (7). In either case, the expression of minimized total costs in (10) does not depend on $\bar{b}$. We summarize our discussion in Proposition 1.

PROPOSITION 1. In the absence of internal corruption, all $\bar{b} \in(0,1)$ can be implemented at the same minimum cost. $w_{\mathrm{A}}^{*}$ and $w_{\mathrm{B}}^{*}$ are thus independent of $\bar{b}$; however, $p^{*}$ should be increased to implement a lower $\bar{b}$.

Note that a lower bound to implementable levels of corruption will be implied if $p$ is bounded from above, i.e., if the principal cannot afford arbitrarily large rewards for successful bribe reports.

We now consider the possibility of internal corruption, the members' cooperation to eliminate the supervisory function of the hierarchy. Existence of mutually beneficial arrangements among the members is clearly a necessary condition for internal corruption. We will assume that it is also sufficient, i.e., that the parties will collude whenever net expected benefits from collusion 
are positive. ${ }^{5}$ There are two occasions in which A and B can collude. The first occasion is before A monitors B, as B can pay A to avoid being monitored. The second arises after B is caught taking the bribe, as B can pay A to avoid being reported. Below we derive the no-collusion constraint and show that A does not collude with $\mathrm{B}$ ex post if he does not ex ante.

Condition (7), used in (6), implies that B's expected utility is $w_{\mathrm{B}}^{*}$. B can safely add $z$ if he can avoid being monitored. The maximum he is willing to pay for this is obviously $z$, which A will reject if

$$
p^{*} \bar{b} \mu\left(m^{*}\right)-c\left(m^{*}\right) \geqslant z .
$$

In the second occasion for collusion, B is caught taking the bribe, hence is willing to pay up to $w_{\mathrm{B}}^{*}+z$ to avoid being reported, which $\mathrm{B}$ rejects if $p \geqslant$ $w_{\mathrm{B}}^{*}+z$. However, as expected, this is already implied by (11). Therefore, (11) is the relevant no-collusion constraint. We investigate below the impact of (11) on the possibility of implementing a $\bar{b} \in(0,1)$.

Proposition 2. The no-collusion constraint is independent of the target level of corruption; either all $\bar{b} \in(0,1)$ can be implemented or none.

Proof. ${ }^{6}$ Substituting (8), (11) can be written as

$$
\mu\left(m^{*}\right) \frac{c^{\prime}\left(m^{*}\right)}{\mu^{\prime}\left(m^{*}\right)}-c\left(m^{*}\right) \geqslant z,
$$

which is independent of $\bar{b}$ because $m^{*}$ is determined by $w_{\mathrm{B}}^{*}$ through (7). Let $M$ be the set of $m$ satisfying (12). $M$ is convex, because the left-hand side of (12) approaches zero as $m \rightarrow 0$, infinity as $m \rightarrow \infty$, and is monotonically increasing in $m$ : we have

$$
\mu^{\prime} \frac{c^{\prime}}{\mu^{\prime}}+\mu\left[\frac{c^{\prime \prime} \mu^{\prime}-c^{\prime} \mu^{\prime \prime}}{\left(\mu^{\prime}\right)^{2}}\right]-c^{\prime}>0
$$

for all $m$, since $\mu^{\prime \prime}<0$. Let $\underline{m}=\min M$; (12) holds with equality for $m^{*}=$ $\underline{m}$. Let also $w_{\mathrm{B}}(\underline{m})$ be the wage that solves $(7)$ for $m=\underline{m}$. Note that $w_{\mathrm{B}}(m)$ is decreasing in $m$. If $w_{\mathrm{B}}(\underline{m})<w$, any equilibrium effort satisfying the nocollusion constraint will violate B's participation constraint. Since this condi-

${ }^{5}$ This is a strong assumption, particularly in large populations of agents, because it implies that collusive agreements can costlessly be negotiated and enforced. High negotiation costs can of course mitigate internal corruption. On the other hand, collusive agreements must be selfenforcing because they are illegal. In most real-world examples, self-enforcingness obtains from the fact that collusive agreements change the parties' threat points. Usually the parties to an organized corruption accumulate evidence that can be used for punishing potential deviators.

${ }^{6} \mathrm{We}$ are grateful to a referee for suggesting the proposition and sketching the arguments in its proof. 
tion does not depend on $\bar{b}$, we conclude that any $\bar{b} \in(0,1)$ can be implemented if and only if $w_{\mathrm{B}}(\underline{m}) \geqslant w$.

Q.E.D.

Proposition 2 is a negative result: given the size of a potential bribe, the reservation wages, and the monitoring technology, it may be impossible to reduce external corruption without generating internal corruption. To eliminate the possibility of internal corruption, the principal must provide A with an expected net reward that is at least equal to the maximum internal bribe $z$ that B may offer A for covering up his corrupt behavior. A's expected net reward from monitoring is increasing in his equilibrium effort $m^{*}$, and exceeds $z$ for $m^{*}>\underline{m}$. In Nash-implementing $\bar{b} \in(0,1)$, the principal can induce A to monitor more intensively by decreasing B's wage, but there is a lower limit $w$ to $w_{\mathrm{B}}$. If an effort $m^{*} \geqslant \underline{m}$ satisfying the no-collusion constraint cannot be induced at the minimum wage $w_{\mathrm{B}}=w$ that satisfies B's participation constraint, the problem admits no solution and the principal cannot implement $\bar{b} \in(0,1)$. Therefore, an implication of Proposition 2 is that the problem of implementing a target level of corruption $\bar{b}$ is less severe in economies where the agents' reservation wages are low and/or the private benefit from corrupt behavior, the bribe, is low.

The following section is devoted to the analysis of hierarchies with three agents. As mentioned in Section II, we shall consider two hierarchies, $H 1$ and $H 2 . H 1$ is the steepest hierarchy with three ranks, whereas $H 2$ is a symmetric pyramidal structure with two ranks.

\section{THE HIERARCHIES $H 1$ AND $H 2$}

The analysis in this section consists of two parts. In the first, we compare the least-cost solutions in the two hierarchies under the assumption that the corresponding no-collusion constraints hold. The relative performance of $H 1$ and $H 2$ then hinges on the level of economies of scale in the detection probability, the behavior of $\mu(n m, n)$ with respect to $n=1,2$. In the second part, we introduce the possibility of internal corruption to see whether and how the results are affected. The focus is on the interplay of economies of scale in the detection probability and the possibility of internal corruption in $H 2$. We also investigate the relative surplus from internal corruption at the top and bottom layers of $H 1$.

\section{The Analysis in the Absence of Internal Corruption}

We consider first the supervision chain $H 1$ where Agent A monitors Agent $\mathrm{B}$ with an effort $m_{\mathrm{A}}$, who in turn monitors Agent $\mathrm{C}$ with an effort $m_{\mathrm{B}}$. The new element in the analysis is thus the presence of a middle-rank agent who monitors and is monitored at the same time. Let $p_{\mathrm{A}}$ and $p_{\mathrm{B}}$ denote the rewards the principal offers for A's and B's successful bribe reports. In the absence 
of internal corruption, the expected utilities and participation constraints of $\mathrm{A}$ and $\mathrm{B}$ are

$$
\begin{gathered}
E U_{\mathrm{A}}=w_{\mathrm{A}}+z+\mu\left(m_{\mathrm{A}}\right) b_{\mathrm{B}} p_{\mathrm{A}}-c\left(m_{\mathrm{A}}\right) \geqslant w \\
E U_{\mathrm{B}}=\left[1-\mu\left(m_{\mathrm{A}}\right)\right] b_{\mathrm{B}}\left(w_{\mathrm{B}}+z+p_{\mathrm{B}} b_{\mathrm{C}} \mu\left(m_{\mathrm{B}}\right)\right) \\
+\left(1-b_{\mathrm{B}}\right)\left(w_{\mathrm{B}}+p_{\mathrm{B}} b_{\mathrm{C}} \mu\left(m_{\mathrm{B}}\right)\right)-c\left(m_{\mathrm{B}}\right) \geqslant w .
\end{gathered}
$$

Agent C's expected utility is as given in (6) where $b=b_{\mathrm{C}}$ and $m=m_{\mathrm{B}}$. Equation (13) displays the impact of A's monitoring on B's objective and incentives. Suppose that B accepts the bribe. Then, his expected income is $w_{\mathrm{B}}+z+p_{\mathrm{B}} b_{\mathrm{C}} \mu\left(m_{\mathrm{B}}\right)$ if he is not caught by $\mathrm{A}$, which happens with probability $1-\mu\left(m_{\mathrm{A}}\right)$. B's income is thus zero with probability $\mu\left(m_{\mathrm{A}}\right) b_{\mathrm{B}}$. If $\mathrm{B}$ is honest, his expected income is $w_{\mathrm{B}}+p_{\mathrm{B}} b_{\mathrm{C}} \mu\left(m_{\mathrm{B}}\right)$. We can now state the principal's problem under $H 1$

$$
\begin{aligned}
& \min \left\{p_{\mathrm{A}} b_{\mathrm{B}}^{*} \mu\left(m_{\mathrm{A}}^{*}\right)+p_{\mathrm{B}} \mu\left(m_{\mathrm{B}}^{*}\right) b_{\mathrm{C}}^{*}\left[1-\mu\left(m_{\mathrm{A}}^{*}\right) b_{\mathrm{B}}^{*}\right]\right. \\
& \left.+w_{\mathrm{A}}+w_{\mathrm{B}}+w_{\mathrm{C}}\right\},
\end{aligned}
$$

subject to

$$
\begin{aligned}
& p_{\mathrm{A}} b_{\mathrm{B}}^{*}=c^{\prime}\left(m_{\mathrm{A}}^{*}\right) / \mu^{\prime}\left(m_{\mathrm{A}}^{*}\right) \\
& \mu\left(m_{\mathrm{A}}^{*}\right)=z /\left[w_{\mathrm{B}}+z+p_{\mathrm{B}} b_{\mathrm{C}}^{*} \mu\left(m_{\mathrm{B}}^{*}\right)\right] \\
& p_{\mathrm{B}} b_{\mathrm{C}}^{*}\left[1-b_{\mathrm{B}}^{*} \mu\left(m_{\mathrm{A}}^{*}\right)\right]=c^{\prime}\left(m_{\mathrm{B}}^{*}\right) / \mu^{\prime}\left(m_{\mathrm{B}}^{*}\right) \\
& \mu\left(m_{\mathrm{B}}^{*}\right)=z /\left(w_{\mathrm{C}}+z\right) \\
& b_{\mathrm{B}}^{*}=b_{\mathrm{C}}^{*}=\bar{b}<1 \\
& E U_{i} \geqslant w, \quad i=\mathrm{A}, \mathrm{B}, \mathrm{C} .
\end{aligned}
$$

The expected cost of rewards in (14) consists of two terms; the first corresponds to the supervision done by A, the second by B. The expected cost of B's supervision looks slightly more complex due to the fact that B will not be rewarded if he is proved corrupt by A; the probability of this event is $\mu\left(m_{\mathrm{A}}^{*}\right) b_{\mathrm{B}}^{*}$. The constraints (15) through (18) are the Nash equilibrium conditions. Equations (16) and (18) state, respectively, B's and C's indifference between accepting and rejecting the bribe. According to (15) and (17), the Nash monitoring efforts $m_{\mathrm{A}}^{*}$ and $m_{\mathrm{B}}^{*}$ must be best replies to the subordinates' bribe acceptance strategies $b_{\mathrm{B}}^{*}$ and $b_{\mathrm{C}}^{*}$. Comparing (15) and (17), we observe that the monitored supervisor's expected marginal net benefit from monitoring is lower.

Note that $w_{\mathrm{A}}$ has no impact on the two monitoring games between $\mathrm{A}$ and $\mathrm{B}$, and B and C. It should thus be chosen to bind A's participation constraint 


$$
w_{\mathrm{A}}^{*}=w-z-\mu\left(m_{\mathrm{A}}^{*}\right) b_{\mathrm{B}}^{*} p_{\mathrm{A}}+c\left(m_{\mathrm{A}}^{*}\right) .
$$

Solving for $w_{\mathrm{B}}$ and $w_{\mathrm{C}}$ from (16) and (18), we obtain

$$
w_{\mathrm{B}}^{*}=z \frac{\left(1-\mu\left(m_{\mathrm{A}}^{*}\right)\right)}{\mu\left(m_{\mathrm{A}}^{*}\right)}-p_{\mathrm{B}} b_{\mathrm{C}}^{*} \mu\left(m_{\mathrm{B}}^{*}\right) \quad \text { and } \quad w_{\mathrm{C}}^{*}=z \frac{\left(1-\mu\left(m_{\mathrm{A}}^{*}\right)\right)}{\mu\left(m_{\mathrm{A}}^{*}\right)} .
$$

Substituting (19) and (20) for $w_{\mathrm{A}}^{*}, w_{\mathrm{B}}^{*}$, and $w_{\mathrm{C}}^{*}$, and using

$$
p_{\mathrm{B}}^{*}=\frac{1}{\bar{b}\left(1-\mu\left(m_{\mathrm{A}}^{*}\right) \bar{b}\right)} \frac{c^{\prime}\left(m_{\mathrm{B}}^{*}\right)}{\mu^{\prime}\left(m_{\mathrm{B}}^{*}\right)}
$$

from (17), the expression of total costs can be written as

$$
\begin{aligned}
& w-z+c\left(m_{\mathrm{A}}^{*}\right)-\frac{\bar{b} \mu\left(m_{\mathrm{A}}^{*}\right) \mu\left(m_{\mathrm{B}}^{*}\right) c^{\prime}\left(m_{\mathrm{B}}^{*}\right)}{\left(1-\mu\left(m_{\mathrm{A}}^{*}\right) \bar{b}\right)} \frac{c^{\prime}\left(m_{\mathrm{B}}^{*}\right)}{\mu^{\prime}\left(m_{\mathrm{B}}^{*}\right)} \\
&+z \frac{\left(1-\mu\left(m_{\mathrm{A}}^{*}\right)\right)}{\mu\left(m_{\mathrm{A}}^{*}\right)}+z \frac{\left(1-\mu\left(m_{\mathrm{B}}^{*}\right)\right)}{\mu\left(m_{\mathrm{B}}^{*}\right)} .
\end{aligned}
$$

The principal's problem is thus reduced to minimizing the expression in (22) with respect to $m_{\mathrm{A}}^{*}$ and $m_{\mathrm{B}}^{*}$ subject to B's and C's participation constraints. $w_{\mathrm{B}}^{*}$ and $w_{\mathrm{C}}^{*}$ will be determined through (20), provided that $E U_{i}\left(w_{i}^{*}\right) \geqslant w$ for $i=\mathrm{B}, \mathrm{C}$. If the $w_{i}^{*}$ that solves (20) yields $E U_{i}\left(w_{i}^{*}\right)<w$, then the wage $w_{i}^{*}$ should be chosen so as to bind the corresponding participation constraint. Proposition 4 characterizes the least cost solution in $H 1$, ignoring the possibility of internal corruption.

Proposition 3. Suppose that $\bar{b} \in(0,1)$ can be implemented. Then the principal chooses $w_{\mathrm{A}}^{*}$ and $w_{\mathrm{C}}^{*}$ as low as possible, so that $E U_{\mathrm{A}}\left(w_{\mathrm{A}}^{*}\right)=$ $E U_{\mathrm{C}}\left(w_{\mathrm{C}}^{*}\right)=w$, whereas $E U_{\mathrm{B}}\left(w_{\mathrm{B}}^{*}\right) \geqslant w$. The optimal rewards $p_{\mathrm{A}}^{*}$ and $p_{\mathrm{B}}^{*}$ are determined through (15) and (21). As in the basic hierarchy, the minimum cost of implementing any $\bar{b} \in(0,1)$ through $H 1$ is independent of $\bar{b}$.

Proof. We have shown that $E U_{\mathrm{A}}\left(w_{\mathrm{A}}^{*}\right)=w$. To show $E U_{\mathrm{C}}\left(w_{\mathrm{C}}^{*}\right)=w$, we consider the two first-order conditions for the problem of minimizing (22). Differentiating with respect to $m_{\mathrm{A}}^{*}$ and $m_{\mathrm{B}}^{*}$ yields two first-order conditions

$$
\begin{aligned}
c^{\prime}\left(m_{\mathrm{A}}^{*}\right)-\frac{\bar{b} \mu\left(m_{\mathrm{B}}^{*}\right)}{\left[1-\mu\left(m_{\mathrm{A}}^{*}\right) \bar{b}\right]} \frac{c^{\prime}\left(m_{\mathrm{B}}^{*}\right)}{\mu^{\prime}\left(m_{\mathrm{B}}^{*}\right)}\left\{\mu^{\prime}\left(m_{\mathrm{A}}^{*}\right)+\frac{\mu\left(m_{\mathrm{A}}^{*}\right) \bar{b} \mu^{\prime}\left(m_{\mathrm{A}}^{*}\right)}{\left[1-\mu\left(m_{\mathrm{A}}^{*}\right) \bar{b}\right]}\right\} \\
-z \frac{\mu^{\prime}\left(m_{\mathrm{A}}^{*}\right)}{\left[\mu\left(m_{\mathrm{A}}^{*}\right)\right]^{2}}=0,
\end{aligned}
$$

and

$$
-\left(\frac{\bar{b} \mu\left(m_{\mathrm{B}}^{*}\right)}{\left[1-\mu\left(m_{\mathrm{A}}^{*}\right) \bar{b}\right]}\right)\left(c^{\prime}\left(m_{\mathrm{B}}^{*}\right)+\mu\left(m_{\mathrm{B}}^{*}\right) \frac{\partial}{\partial m_{\mathrm{B}}}\left[\frac{c^{\prime}\left(m_{\mathrm{B}}^{*}\right)}{\mu^{\prime}\left(m_{\mathrm{B}}^{*}\right)}\right]\right)-z \frac{\mu^{\prime}\left(m_{\mathrm{B}}^{*}\right)}{\left[\mu\left(m_{\mathrm{B}}^{*}\right)\right]^{2}}<0 .
$$


The expression above is always negative because $\left[\partial / \partial m_{\mathrm{B}}\right]\left[c^{\prime}\left(m_{\mathrm{B}}\right) / \mu^{\prime}\left(m_{\mathrm{B}}\right)\right]>$ 0 . Therefore, total costs can be reduced by inducing higher effort on $\mathrm{B}$. This can be done by choosing the lowest possible $w_{\mathrm{C}}$, see (20), which implies that $w_{\mathrm{C}}^{*}=w$. Given the corresponding $m_{\mathrm{B}}^{*}$ through (18), the value of $m_{\mathrm{A}}^{*}$ is determined through the first-order condition in (23), and the resulting wage $w_{\mathrm{B}}^{*}$ that solves (16) may well be an interior solution, leaving $\mathrm{B}$ a surplus. If this $w_{\mathrm{B}}^{*}$ violates B's participation constraint, then $w_{\mathrm{B}}^{*}$ is given by $E U_{\mathrm{B}}\left(w_{\mathrm{B}}^{*}\right)$ $=w$. Finally, the expression in (22) is clearly independent of $\bar{b}$; hence if all $\bar{b} \in(0,1)$ can be implemented, they can be implemented at the same minimum cost.

Q.E.D.

Consider the problem statement in (14) through (18) to gain some intuition for why the principal chooses $w_{\mathrm{C}}^{*}$ as low as possible, while $w_{\mathrm{B}}^{*}$ does not necessarily bind $E U_{\mathrm{B}}$. Reductions in $w_{\mathrm{B}}$ and $w_{\mathrm{C}}$ have asymmetric effects on equilibrium monitoring efforts: to decrease $w_{\mathrm{B}}$ and keep corruption at its target level $\bar{b}$ the principal must induce a higher $m_{\mathrm{A}}^{*}$. To decrease $w_{\mathrm{C}}$ he must similarly induce a higher $m_{\mathrm{B}}^{*}$, but on the other hand he can induce a lower $m_{\mathrm{A}}^{*}$, as required by (16). Reductions in $w_{\mathrm{C}}$ thus allow the principal to economize on $w_{\mathrm{A}}+p_{\mathrm{A}} \bar{b} \mu\left(m_{\mathrm{A}}^{*}\right)$ because $c\left(m_{\mathrm{A}}^{*}\right)$ will be lower. This additional effect arises thanks to the impact or externality of A's monitoring on B's monitoring incentives and implies that reductions in $w_{\mathrm{C}}$ generate larger cost savings than reductions in $w_{\mathrm{B}}$, yielding the result $w_{\mathrm{C}}^{*}=w$. Using this fact, we can express the minimum cost in $H 1$ as

$$
T C(H 1)=2 w-z+c\left(m_{\mathrm{A}}^{*}\right)-p_{\mathrm{B}}^{*} \mu\left(m_{\mathrm{B}}^{*}\right) \mu\left(m_{\mathrm{A}}^{*}\right) \bar{b}^{2}+z \frac{\left(1-\mu\left(m_{\mathrm{A}}^{*}\right)\right)}{\mu\left(m_{\mathrm{A}}^{*}\right)} .
$$

In minimizing costs, the principal actually exploits the externality that goes from A's monitoring to $\mathrm{B}$. The term $-p_{\mathrm{B}}^{*} \mu\left(m_{\mathrm{A}}^{*}\right) \mu\left(m_{\mathrm{B}}^{*}\right) \bar{b}^{2}$ in (24) represents the expected reduction in the principal's reward payments to $\mathrm{B}$ that stems from the fact that B will not be rewarded if he himself is proved corrupt.

We now proceed with the principal's problem under $H 2$. The hierarchies we considered so far assign one subordinate to each supervisor. In $H 2$, one supervisor (A) monitors two agents (B and C), and the per-subordinate detection probability is denoted $\mu(2 m, 2)$ where $m$ is the per-subordinate monitoring effort. We say that the detection probability exhibits economies of scale if $\mu(2 m, 2)>\mu(m, 1)$ for all $m$, that is, if the per-subordinate detection probability is uniformly higher under two subordinates than one.

We consider symmetric Nash equilibria of $H 2$ where A monitors each subordinate with the same effort $m$, generating a total monitoring cost of $c(2 m)$. Let $p$ be the reward per successful bribe report. Ignoring the possibility of internal corruption, the expression of total costs is $p \mu\left(2 m^{*}, 2\right)\left[b_{\mathrm{B}}^{*}+b_{\mathrm{C}}^{*}\right]$ $+w_{\mathrm{A}}+w_{\mathrm{B}}+w_{\mathrm{C}}$, which the principal minimizes choosing $p, w_{\mathrm{A}}, w_{\mathrm{B}}$ and $w_{\mathrm{C}}$ subject to the constraints 


$$
\begin{aligned}
& \mu\left(2 m^{*}, 2\right)=z /\left(z+w_{i}\right), \quad i=\mathrm{B}, \mathrm{C} \\
& p b_{i}^{*}=c^{\prime}\left(2 m^{*}\right) / \mu^{\prime}\left(2 m^{*}, 2\right), \quad i=\mathrm{B}, \mathrm{C}, \\
& b_{\mathrm{B}}^{*}=b_{\mathrm{C}}^{*}=\bar{b}<1, \\
& E U_{i} \geqslant w, \quad i=\mathrm{A}, \mathrm{B}, \mathrm{C} .
\end{aligned}
$$

The expected utilities of $\mathrm{B}$ and $\mathrm{C}$ are of the form given in (6), and A's expected utility is $E U_{\mathrm{A}}=w_{\mathrm{A}}+z+p_{\mathrm{A}} \mu(2 m, 2)\left[b_{\mathrm{B}}+b_{\mathrm{C}}\right]-c(2 m)$. Using (25) to substitute for $w_{i}$ and the fact that A's participation constraint should be binding, we can express total costs as

$$
T C(H 2)=w-z+c\left(2 m^{*}\right)+2 z \frac{1-\mu\left(2 m^{*}, 2\right)}{\mu\left(2 m^{*}, 2\right)},
$$

where $m^{*}$ minimizes (27) subject to the participation constraints of B and C. $m^{*}$ should thus satisfy the first-order condition

$$
c^{\prime}\left(2 m^{*}\right)=z \frac{\mu^{\prime}\left(2 m^{*}, 2\right)}{\left[\mu\left(2 m^{*}, 2\right)\right]^{2}}
$$

if the wages $w_{i}\left(m^{*}\right)$ that solve the equilibrium conditions in (25) satisfy the participation constraints

$$
w_{i}^{*}\left(m^{*}\right)=z \frac{1-\mu\left(2 m^{*}, 2\right)}{\mu\left(2 m^{*}, 2\right)} \geqslant w, \quad i=\mathrm{B}, \mathrm{C} .
$$

If $w_{i}^{*}\left(m^{*}\right)<w$, then the least cost solution is obtained by substituting $w_{i}^{*}$ $=w$ and the $m^{*}$ that solves (25) into the principal's objective. We can now evaluate the impact of economies of scale in $\mu(2 m, 2)$ on $T C(H 2)$.

PROPOSITION 4. If economies of scale in the detection probability are suffciently large, then $T C(H 2)<T C(H 1)$.

Proof. Consider an increase in the economies of scale in the detection probability and suppose that in $H 2$ the principal keeps $w_{\mathrm{B}}$ and $w_{\mathrm{C}}$ constant at their initial levels. Since $\mu(2 m, 2)$ is now higher, $m$ should be decreased to keep (25) holding. Hence, $T C=w-z+c\left(2 m^{*}\right)+w_{\mathrm{B}}+w_{\mathrm{C}}$ must decrease. Notice that implementation costs may decrease further if wages are adjusted in response to the increase in $\mu(2 m, 2)$. Clearly, if economies of scale are sufficiently large, $T C(H 2)$ will fall below $T C(H 1)$.

Q.E.D.

Implementation costs in $H 2$ will be lower than in $H 1$ if the per-subordinate detection probability $\mu(2 m, 2)$ is sufficiently higher than $\mu(m, 1)$. Just how large these economies of scale in monitoring should be depends on the curvatures of $\mu(2 m, 2)$ and $c(2 m)$. Though at this level of generality the critical 
level of economies of scale is impossible to determine, economies of scale in the detection probability is a necessary condition for $T C(H 2)<T C(H 1){ }^{7}$

\section{Introducing Internal Corruption}

We now introduce the possibility of internal corruption. In $\mathrm{H} 2$, the nocollusion constraint is

$$
2 p^{*} \mu\left(2 m^{*}, 2\right) \bar{b}-c\left(2 m^{*}\right) \geqslant 2 z
$$

On the left-hand side we have the supervisor's expected net benefit from monitoring; on the right-hand side $2 z$ represents the subordinates' (B and C) maximum willingness to pay to avoid being monitored. If (30) does not hold at the least-cost solution, then the principal cannot implement $\bar{b}{ }^{8}$

Consider now the supervision chain $H 1$. The monitored agents $\mathrm{B}$ and $\mathrm{C}$ are each willing to pay his supervisor up to $z$ for safely accepting bribes. A will reject B's offer if

$$
\mu\left(m_{\mathrm{A}}^{*}\right) \bar{b} p_{\mathrm{A}}^{*}-c\left(m_{\mathrm{A}}^{*}\right) \geqslant z,
$$

and similarly B will reject C's offer if

$$
\left[1-\mu\left(m_{\mathrm{A}}^{*}\right) \bar{b}\right] p_{\mathrm{B}}^{*} \bar{b} \mu\left(m_{\mathrm{B}}^{*}\right)-c\left(m_{\mathrm{B}}^{*}\right) \geqslant z .
$$

Note that $\mathrm{B}$ must be implied in any collusion because he has monitoring relations with the other two agents. The question of interest is whether it is easier for B to collude with A or C at the least cost solution. Which part of the supervision chain generates a larger surplus from internal corruption?

PROPOSITION 5. The relevant no-collusion constraint for the principal in implementing the least cost solution in $H 1$ is (31): The surplus from internal corruption in the upper part of the supervision chain (between $A$ and $B$ ) is larger than in its lower part (between $B$ and $C$ ).

Proof. Substituting the equilibrium conditions, (31) and (32) can be written as

${ }^{7}$ It can be shown that $T C(H 2)>T C(H 1)$ if $\mu(2 m, 2) \leqslant \mu(m, 1) . H 1$ has two advantages. The first is that $H 1$ displays a monitoring externality, the fact that $\mathrm{B}$ will not be rewarded for detecting $\mathrm{C}$ and receives a utility zero if he is detected by $\mathrm{A}$, which the principal can exploit to reduce expected costs. Second, even if we remove this externality and let B be paid a reward for his successful bribe report in all circumstances, the fact that $\mu(2 m, 2) \leqslant \mu(m)$ generates cost savings in $H 1$ for two reasons: (i) due to diseconomies of scale in $\mu$, the target level of corruption can be implemented through lower levels of effort in $H 1$, and (ii) since $c(2 m)>2 c(m)$ by strict convexity of $c(\cdot)$, the total cost of effort to the supervisors will certainly be lower under $H 1$, which can generate further savings on the wage bill.

${ }^{8}$ As mentioned in Proposition 2, the no-collusion constraint is independent of the target level of corruption. This result holds in all hierarchies; in the case of $H 2$, it can easily be verified using the equilibrium conditions. 
$\mu\left(m_{\mathrm{A}}^{*}\right) \frac{c^{\prime}\left(m_{\mathrm{A}}^{*}\right)}{\mu^{\prime}\left(m_{\mathrm{A}}^{*}\right)}-c\left(m_{\mathrm{A}}^{*}\right) \geqslant z \quad$ and $\quad \mu\left(m_{\mathrm{B}}^{*}\right) \frac{c^{\prime}\left(m_{\mathrm{B}}^{*}\right)}{\mu^{\prime}\left(m_{\mathrm{B}}^{*}\right)}-c\left(m_{\mathrm{B}}^{*}\right) \geqslant z$.

Since the expressions on the left-hand sides of the constraints in (33) are monotonically increasing in $m$ (see the proof of Proposition 2), to establish the result we need to show that $m_{\mathrm{A}}^{*}<m_{\mathrm{B}}^{*}$. We have shown that $w_{\mathrm{C}}^{*}=w$; hence $m_{\mathrm{B}}^{*}$ is determined through the equilibrium condition $\mu\left(m_{\mathrm{B}}^{*}\right)=z /(z+$ $w) . m_{\mathrm{A}}^{*}$, on the other hand, satisfies $\mu\left(m_{\mathrm{A}}^{*}\right)=z /\left(w_{\mathrm{B}}^{*}+z+p_{\mathrm{B}}^{*} \bar{b} \mu\left(m_{\mathrm{B}}^{*}\right)\right)$, which shows that $m_{\mathrm{A}}^{*}$ must be higher if $w_{\mathrm{B}}^{*}$ is reduced. Consider the lowest possible $w_{\mathrm{B}}^{*}$ binding B's participation constraint: $\underline{w}_{\mathrm{B}}=w-p_{\mathrm{B}}^{*} \bar{b} \mu\left(m_{\mathrm{B}}^{*}\right)+c\left(m_{\mathrm{B}}^{*}\right)$. Clearly, the highest possible $m_{\mathrm{A}}^{*}$ obtains for $w_{\mathrm{B}}^{*}=\underline{w}_{\mathrm{B}}$. Now, even this highest possible $m_{\mathrm{A}}^{*}$ must be lower than $m_{\mathrm{B}}^{*}$, as $\mu\left(m_{\mathrm{A}}^{*}\right)=z /\left(w+z+c\left(m_{\mathrm{B}}^{*}\right)\right)<z /(w$ $+z)=\mu\left(m_{\mathrm{B}}^{*}\right)$. This completes the proof.

Q.E.D.

The result in Proposition 5 is implied by two facts: First, the surplus from collusion is low when the supervisor's expected benefit from monitoring is high, which obtains if the supervisor is induced to exert a high effort in the Nash equilibrium. The second fact is that the principal in implementing the least-cost solution finds it cheaper to induce effort on supervisor B for reasons given in the discussion of Proposition 3. The two supervisors A and B can each be bribed $z$, but B's expected benefit from monitoring is higher than A's; hence the expected surplus from collusion between $B$ and $C$ is lower than the corresponding surplus between $\mathrm{A}$ and $\mathrm{B}$. The result will hold even more strongly under the assumption that supervisors can receive larger external bribes than can their subordinates.

We can now proceed to compare the no-collusion constraints in $H 1$ and $H 2$. We have shown in Proposition 4 that $H 2$ will implement the target level of corruption at a lower cost if $\mu(2 m, 2)$ exhibits sufficient economies of scale. This brings in the question as to whether economies of scale, which favor the choice of $\mathrm{H} 2$, expose the principal to a higher cost of internal corruption.

The no-collusion constraints (31) in $H 1$ and (30) in $H 2$ can be written as $\mu\left(m_{\mathrm{A}}^{*}\right) \frac{c^{\prime}\left(m_{\mathrm{A}}^{*}\right)}{\mu^{\prime}\left(m_{\mathrm{A}}^{*}\right)}-c\left(m_{\mathrm{A}}^{*}\right) \geqslant z \quad$ and $\quad \mu\left(2 m^{*}, 2\right) \frac{c^{\prime}\left(2 m^{*}\right)}{\mu^{\prime}\left(2 m^{*}, 2\right)}-\frac{c\left(2 m^{*}\right)}{2} \geqslant z$.

The surplus from collusion in $H 2$ is thus larger if

$$
\mu\left(m_{\mathrm{A}}^{*}\right) \frac{c^{\prime}\left(m_{\mathrm{A}}^{*}\right)}{\mu^{\prime}\left(m_{\mathrm{A}}^{*}\right)}-c\left(m_{\mathrm{A}}^{*}\right)>\mu\left(2 m^{*}, 2\right) \frac{c^{\prime}\left(2 m^{*}\right)}{\mu^{\prime}\left(2 m^{*}, 2\right)}-\frac{c\left(2 m^{*}\right)}{2},
$$

where the cost-minimizing efforts $m^{*}$ and $m_{\mathrm{A}}^{*}$ are determined, respectively, through (28) and (23). Whether (34) holds depends on the relationship between $\mu(2 m, 2)$ and $\mu(m)$ (including their derivatives), on the curvature of $c(\cdot)$, and on the level of cost-minimizing efforts $m^{*}$ and $m_{\mathrm{A}}^{*}$ in $H 2$ and $H 1$. 
At the present level of generality, the definition of economies of scale as $\mu(2 m, 2)>\mu(m, 1)$ is of little help for assessing the relative surplus from internal corruption. We shall accordingly investigate (34) under additional assumptions about the type of scale economies and the properties of $c(\cdot)$. We consider below the case of constant returns to scale.

Proposition 6. Assume constant returns to scale in the detection probability and a linear cost-of-effort function. Then, the expected surplus from internal corruption is larger in $H 2$ than in $H 1$.

Proof. Under the assumptions stated in the proposition, $c(m)=\mathrm{cm}$ and $\mu(2 m, 2)=\mu(m)$ where $c$ is the constant marginal cost. Equation (34) can thus be written as

$$
\mu\left(m_{\mathrm{A}}^{*}\right) \frac{c}{\mu^{\prime}\left(m_{\mathrm{A}}^{*}\right)}-c m_{\mathrm{A}}^{*}>\mu\left(m^{*}\right) \frac{c}{\mu^{\prime}\left(m^{*}\right)}-c m^{*},
$$

which holds if and only if $m_{\mathrm{A}}^{*}>m^{*}$. Consider now the corresponding firstorder conditions (23) and (28) determining $m_{\mathrm{A}}^{*}$ and $m^{*}$,

$$
c=z \frac{\mu^{\prime}\left(m_{\mathrm{A}}^{*}\right)}{\left[\mu\left(m_{\mathrm{A}}^{*}\right)\right]^{2}}+X \quad \text { and } \quad c=z \frac{\mu^{\prime}\left(m^{*}\right)}{\left[\mu\left(m^{*}\right)\right]^{2}},
$$

where $X>0$. Note that these conditions imply $m_{\mathrm{A}}^{*}>m^{*}$. Hence (35) holds.

Q.E.D.

If the cost-of-effort function is strictly convex, the condition in (34) depends on the curvature of $c(\cdot)$ in a complex way; however, we should expect (34) holding under constant returns to scale in $\mu(2 m, 2)$ if $c(\cdot)$ is not too convex.

What is the relationship between economies of scale and the expected surplus from internal corruption in $H 2$ ? The answer depends on which of the two opposing forces, explained below, dominates the other. When, at the initial cost-minimizing level of effort, larger economies of scale imply an increase in $\mu / \mu^{\prime}$, the right-hand side of (34) increases, but higher $\mu / \mu^{\prime}$ implies, through (28), that the principal will implement a lower effort, which, in turn, will reduce the right-hand side of (34). The direction of these forces is reversed when economies of scale imply a lower $\mu / \mu^{\prime}$ at the initial level of effort; then the principal will implement a higher effort, which tends to offset the impact of a lower $\mu / \mu^{\prime}$ on the no-collusion constraint. Thus, larger economies of scale will increase the surplus from internal corruption if the direct effect of economies of scale on the no-collusion constraint is dominated by the opposing effect generated by cost-minimizing adjustments in the supervisor's effort. Without further assumptions about the functional transformation of $\mu(2 m, 2)$, it is impossible to predict the impact of larger economies of scale on the no-collusion constraint in $H 2$. Let us consider a linear transformation $\mu(2 m, 2) \rightarrow \hat{\mu}(2 m, 2)=\alpha \mu(2 m, 2)$ where $\alpha<1$. This corresponds to disecono- 
mies of scale where the new per-subordinate probability of success is a fraction $\alpha$ of the old, keeping the ratio $\mu / \mu^{\prime}$ unchanged. To see what happens to the no-collusion constraint in $H 2$, we have to determine whether the principal implements a higher effort under $\hat{\mu}$. Let $m^{*}$ denote the cost-minimizing effort per subordinate under $\mu$. Inspecting (28) reveals that $c^{\prime}\left(2 m^{*}\right)<z \hat{\mu}^{\prime}\left(2 m^{*}, 2\right) /$ $\left[\hat{\mu}\left(2 m^{*}, 2\right)\right]^{2}$; hence to restore equality the principal will implement a higher effort $\hat{m}>m^{*}$ under the detection probability $\hat{\mu}$. But then, by monotonicity of the no-collusion constraint and using $\hat{\mu}=\alpha \mu$,

$$
\alpha \mu(2 \hat{m}, 2) \frac{c^{\prime}(2 \hat{m})}{\alpha \mu^{\prime}(2 \hat{m}, 2)}-c(2 \hat{m})>\mu\left(2 m^{*}, 2\right) \frac{c^{\prime}\left(2 m^{*}\right)}{\mu^{\prime}\left(2 m^{*}, 2\right)}-c\left(2 m^{*}\right) .
$$

We have proved the following:

Proposition 7. Consider two per-subordinate detection probabilities $\mu$ and $\hat{\mu}$ such that $\hat{\mu}=\alpha \mu$ with $\alpha<1$. The surplus from internal corruption in $\mathrm{H} 2$ is lower under $\hat{\mu}$ than under $\mu$.

Proposition 7 can be generalized to any transformation $\mu \rightarrow \hat{\mu}$ such that $\mu /$ $\mu^{\prime}=\hat{\mu} / \hat{\mu}^{\prime}$. Then, if $\hat{\mu}>\mu$, the first-order condition (28) implies that the cost-minimizing effort is lower under $\hat{\mu}$, and monotonicity of the no-collusion constraint implies that the expected collusion surplus is larger under $\hat{\mu}$. This inverse relationship between the supervisor's expected rewards from monitoring and economies of scale implies the following: Though ignoring the nocollusion constraints yields $T C(H 2)<T C(H 1)$ under large economies of scale in monitoring, $H 2$ may not dominate $H 1$ if the possibility of internal corruption is taken into account.

\section{SUMMARY AND EXTENSIONS}

This paper provides a formal analysis of the relationship between the structure of monitoring hierarchies and corruption. The model has three agents and a principal who faces the problem of designing a hierarchy, a reward scheme for supervisors, and wages for the agents to minimize the cost of implementing a target level of corruption. Each agent makes a binary choice on whether to accept a bribe (external corruption); supervisors determine in addition an effort to monitor their subordinates. The principal's problem is complicated by the possibility of internal corruption, the agents' cooperation to organize corruption and to share the corresponding private surplus. The results are summarized below.

(1) Inducing monitoring to keep external corruption under control at a target level brings in the possibility of internal corruption. Depending on the monitoring technology, the agents' reservation wages, and potential bribes, the constraint of internal corruption may deny any solution to the implementation 
problem, leaving only the full corruption outcome implementable. In that case the principal's only remaining option is to improve the monitoring technology.

(2) Though supervision is more effective on supervisors, the surplus from internal corruption is larger in the upper part than in the lower part of the supervision chain $H 1$. Here the principal faces a trade-off: exploiting the impact of the top supervisor's monitoring on the middle rank supervisor to minimize implementation costs brings about a larger surplus from collusion between the two supervisors.

(3) The relative performance of the hierarchy $\mathrm{H} 2$ where one supervisor monitors two agents depends on the monitoring technology. If the detection probability exhibits sufficiently large economies of scale, in the sense that the per-subordinate probability of detection is sufficiently higher under two subordinates than one, the flat hierarchy $\mathrm{H} 2$ is more efficient in reducing external corruption than the steep hierarchy $H 1$. Whether larger economies of scale, which favor $H 2$, actually increase the expected surplus from internal corruption depends on the forms of detection probability and cost-of-effort functions. The answer is affirmative if the least-cost implementation effort is sensitive to changes in economies of scale. We show that this is the case for proportional shifts in the detection probabilities, a linear transformation $\mu \rightarrow$ $\hat{\mu}$ where $\hat{\mu}=\alpha \mu$ with $\alpha<1$. When this relationship obtains, we can no longer conclude that $H 2$ dominates $H 1$ under large economies of scale in monitoring.

In this paper we focused exclusively on the supervisory function of hierarchies in confronting corruption, external and internal. There are many other functions of hierarchies and bureaucracies with which we have not dealt here, but which we believe should be incorporated into our framework, and which we hope to address in the future. Most important among these functions are gathering and processing of information. Furthermore, several hierarchical layers may be implied in a decision where the higher layers do selective reviewing, which brings in the possibility of monitoring several layers down the hierarchy. We have ignored these possibilities in this paper. Another important extension is to incorporate a formal model of the market in which the agents operate; for example, competition for bribes among bottom-level agents may decrease the equilibrium size of the bribe on the one hand, and increase the benefits from collusion, on the other. Note also that the number of agents in a given hierarchical layer may influence the state of competition for bribes at that layer.

\section{REFERENCES}

Baron, D. P., and Besanko, D., “Information, Control and Organizational Structure.” J. Econom. Management Strategy 1, 2:237-275, 1992.

Bolton, Patrick, and Dewatripont, M., "The Firm as a Communication Network,' Quart. J. Econom. 109, 4:809-839, 1994. 
Calvo, Guilermo, “The Economics of Supervision." In H. Nalbantian, Ed., Incentives, Cooperation and Risk Sharing, pp. 87-103. New Jersey: Rowman \& Littlefield, 1987.

Calvo, Guilermo, and Wellisz, Stanislaw, "Supervision, Loss of Control, and the Optimal Size of the Firm.' J. Polit. Econom. 86, 5:943-952, 1978.

Cyert, Richard, and March, James, A Behavioral Theory of the Firm. Englewood Cliffs, NJ: Prentice-Hall, 1963.

Kofman, F., and Lawarrée, J., “Collusion in Hierarchical Agency,' Econometrica 61, 3:629656, 1993.

Laffont, Jean Jacques, “Analysis of Hidden Gaming in a Three Level Hierarchy," J. Law Econom. Organiz. 6, 3:301-324, 1990.

Radner, Roy, "The Organization of Decentralized Information Processing." Econometrica 61, 6:1109-1146, 1993.

Tirole, Jean, "Hierarchies and Bureaucracies: On the Role of Collusion in Organizations.' J. Law Econom. Organiz. 2, 2:181-214, 1986.

Tirole, Jean, “The Multicontract Organization."' Canad. J. Econom. 21, 3:459-466, 1988.

Varian, Hal R., 'Monitoring Agents with Other Agents.' J. Inst. Theoret. Econom. 146, 2:153174, 1990.

Williamson, Oliver, "Hierarchical Control and the Optimal Firm Size.' J. Polit. Econom. 75, 2:123-138, 1967. 